\title{
Complications in Neuroanesthesia
}

\author{
Hemanshu Prabhaker (editor). Elsevier, 2016, 502 pages. ISBN 978-0-12-804075-1
}

\author{
Melissa Brockerville, MD • Lashmi Venkatraghavan, MD
}

Received: 2 August 2016/Accepted: 11 August 2016/Published online: 16 August 2016

(C) Canadian Anesthesiologists' Society 2016

The textbook Complications in Neuroanesthesia is a unique addition to the literature in that it provides a review of complications that may occur during the perioperative management of neurosurgical and neurologic patients. The book is divided into ten sections: Complications Related to the Brain, Complications Related to Spinal Cord, Complications Related to Cardio-vascular System, Complications Related to Coagulation, Complications Related to Respiratory System, Complications Related to Airway, Complications Related to Fluid and Electrolyte Disturbances, Postoperative Pain, Miscellaneous, and Clinical Scenarios. Each section is divided into several chapters (1-11 chapters per section) that focus on specific complications. The chapters themselves follow a uniformly organized format, with a discussion of the pathophysiology, signs/symptoms, diagnosis, treatment, and prevention of the specific complication, if applicable.

On the surface, the textbook is somewhat daunting at 502 pages. The chapters, however, are easy to read and understand, and complex concepts are comprehensively explained, with numerous diagrams, images, and tables complementing the text. The book's up-to-date, thorough referencing is an additional strength.

The textbook reviews all of the major complications, with no major topic missed or incompletely covered. Some of the more rare complications, such as postoperative visual loss and the hazards of advanced neuromonitoring, were described very well. There were some complications reviewed,

M. Brockerville, MD $\cdot$ L. Venkatraghavan, MD $(\bowtie)$

Department of Anesthesia and Pain Management, Toronto

Western Hospital, University of Toronto, Toronto, ON, Canada

e-mail: lashmi.venkatraghavan@uhn.on.ca however, that were not necessarily specific to neurosurgical anesthesia (i.e., hypercapnia, hypoxemia, coagulopathy, hemodynamic instability, thromboembolism, stridor, anaphylaxis, hypothermia). In most cases, though, the authors attempted to make specific application and reference to the neurosurgical setting. For example, in the chapter on hemodynamic instability, the authors reviewed reasons for its occurrence that should be specifically considered in the neurosurgical setting. Some chapters provide excessive detail about the diagnosis and treatment of certain complications (i.e., thromboembolism, autonomic disturbances). Similarly, there are two chapters dedicated to postoperative pain management after intracranial and spine surgery, which arguably may not be relevant to this book addressing "complications."

Despite being a multi-authored book, with the majority of the authors not being from North American centers, the practice recommendations are, for the most part, consistent with Canadian and North American practice. Specifically, the strong referencing allows the reader to determine easily the origin of the specific recommendation if required. Although there are a few grammatical errors scattered throughout the text, it does not affect the reader's ability to read and interpret the text.

Ten clinical scenarios are provided at the end of the textbook, in each of which a specific clinical scenario is presented and the diagnosis and subsequent management reviewed. These scenarios allow the practitioner to apply information gained from this book to specific clinical practice settings. That said, the scenarios are presented in a somewhat random order, and it would have been better if they had been presented immediately following the relevant chapters. 
In summary, this textbook is a comprehensive, well-organized, up-to-date review of complications that may occur in neurosurgical and neurologic patients. As it is the only textbook to provide such a comprehensive overview of the complications specifically related to neuroanesthesia, we would recommend this book to any trainee or consultant neuroanesthesiologist, intensivist, or neurosurgeon.
Conflicts of interest The authors have no conflict of interest to disclose.

Editorial responsibility This submission was handled by Dr. Hilary P. Grocott, Editor-in-Chief, Canadian Journal of Anesthesia. 